\title{
Hemophagocytic Lymphohistiocytosis/Macrophage-Activation Syndrome (HLH/MAS) Following Treatment with Tisagenlecleucel
}

\author{
Reyes Maria Martin-Rojas ${ }^{1}$, Ignacio Gomez-Centurion ${ }^{1}$, Rebeca Bailen ${ }^{1}$, Mariana Bastos ${ }^{1}$, \\ Francisco Diaz-Crespo ${ }^{1}$, Diego Carbonell ${ }^{1}$, Rafael Correa-Rocha ${ }^{2}$, Marjorie Pion ${ }^{2}$, Cristina \\ Muñoz $^{1}$, Milagros Sancho ${ }^{1}$, Isabel Gomez-Fernandez ${ }^{1}$, Gillen Oarbeascoa ${ }^{1}$, Ana \\ Perez-Corral $^{1}$, Carolina Martinez-Laperche ${ }^{1}$, Javier Anguita ${ }^{1}$, Ismael Buño ${ }^{1}$, Javier \\ Menarguez $^{1}$, Jose Luis Diez-Martin ${ }^{1}$, and Mi Kwon ${ }^{3}$ \\ ${ }^{1}$ Hospital General Universitario Gregorio Marañón \\ ${ }^{2}$ Instituto de Investigación Sanitaria Gregorio Marañón \\ ${ }^{3}$ Hospital General Universitario Gregorio Maranon
}

August 8, 2021

\begin{abstract}
CAR-T cell related HLH/MAS is an unusual manifestation of severe cytokine release syndrome (CRS) with high mortality rates and a challenging diagnosis. The establishment of specific diagnosis criteria is essential, and the combination of several techniques for CAR-T cell follow-up, allows a more precise management of this complication.
\end{abstract}

\section{INTRODUCTION}

Immunotherapy with T-cells genetically engineered to express CD19-specific chimeric antigen receptor (CAR) has dramatically changed the treatment of aggressive B-cell malignancies ${ }^{1,2}$. Two products- Tisagenlecleucel and Axicabtagene ciloleucel- have been approved by the European Medicines Agency (EMA) for the treatment of relapsed/refractory CD19+ diseases. Tisagenlecleucel (Tisa-Cel) is a CD19-targeted CAR-T cell therapy approved to treat adult patients with relapsed/refractory diffuse large B-cell lymphoma (DLBCL) after two or more lines of systemic therapy and children and adults up to age 25 with relapsed/refractory B-cell acute lymphoblastic leukemia (ALL) ${ }^{3,4}$.

Despite CAR-T cells can induce rapid and durable responses, this therapy is associated to specific and severe toxicities, representing an obstacle in its widespread use. Cytokine-release syndrome (CRS) is the most frequent toxicity after infusion ${ }^{5,6}$. This systemic inflammatory response can rarely evolve into a fulminant hemophagocytic lymphohistiocytosis/macrophage-activation syndrome (HLH/MAS) which is associated with high mortality rates ${ }^{7,8}$. Diagnosis criteria for this entity have been recently proposed ${ }^{7}$, but discrimination between severe CRS, CAR-T related HLH/MAS and malignancy-associated HLH/MAS can be challenging.

We describe two cases of CAR-T cell related HLH/MAS and the difficulties linked to the diagnosis and management of this unusual complication.

\section{CASES REPORT}

\subsection{CASE 1}

A 34-year-old male with refractory T-cell rich DLBCL with associated HLH/MAS at diagnosis, underwent Tisa-Cel therapy in 2019. High tumor burden with progressive disease was documented after bridging therapy. 
During lymphodepletion with cyclophosphamide and fludarabine, persistent fever with neutropenia was treated with empiric broad-spectrum antimicrobials agents, without resolution.

On day +1 , he presented with grade 1 CRS, managed with tocilizumab on days +15 and +20 , achieving partial response. However, high grade fever followed with hyperferritinemia (peak $50,000 \mathrm{ng} / \mathrm{ml}$ on day +23 ), severe cytopenias, altered liver function, coagulopathy and high values of IL-15, IL-1 $\beta$, GM-CSF and IL-6. HLH/MAS was suspected and bone marrow aspiration was performed on day +22 , showing hemophagocytosis and no evidence of infiltration by lymphoma.

In order to distinguish between CAR-T cell related and malignancy associated HLH/MAS, a PET-CT was performed on day +25 , showing paradoxical response. Concurrently, CAR-T cells expansion in peripheral blood (PB) was detected by flow cytometry and polymerase chain reaction (PCR). Furthermore, a lymph node biopsy was performed where CAR-T cells were detected, with only $4 \%$ of neoplastic lymphocytes (Figure 1).

Subsequently, high dose corticosteroids, siltuximab, anakinra and cyclophosphamide were administered. Hemoadsorption with extracorporeal cytokine adsorber $\left(\right.$ CytoSorb $\left.^{\circledR}\right)$ was initiated on day +35 . Etoposide was not considered due to severe liver function impairment.

Patient showed no response to treatment, and died from multiorgan failure on day +36 . Tumor necrosis was the predominant finding in necropsy, with an estimated $15 \%$ of residual tumor.

\subsection{CASE 2}

A 22-year old male with relapsed/refractory Philadelphia chromosome negative B-cell ALL underwent Tisacel therapy in 2020. After a bridging therapy with cyclophosphamide, vincristine and dexamethasone, he received lymphodepletion with fludarabine and cyclophosphamide. At the time of Tisa-cel infusion the patient was afebrile with a baseline ferritin of $6905 \mathrm{ng} / \mathrm{mL}$. He developed a grade 1 CRS six hours after infusion, progressing to grade 2 on day +2 , and was treated with three doses of tocilizumab. Plasma cytokine levels showed high values of IL-15, GM-CSF and IL-6. Both symptoms and cytokine levels rapidly improved after administration of tocilizumab.

On day +11 he presented with a new episode of fever, high flow oxygen therapy requirements, impaired renal and liver function, increased ferritin levels (peak $800,000 \mathrm{ng} / \mathrm{mL}$ on day +14 ) and coagulopathy. CAR-T cells expansion in $\mathrm{PB}$ was detected $(4,141 \mathrm{CAR}-\mathrm{T} / \mathrm{mL}$ on day +14$)$. At this point, the patient met criteria of CAR-T related HLH/MAS and further studies were performed, showing high levels of soluble CD25 (>10,000 pg/mL), IL-18 (>10,000 pg/mL), INF-gamma $(1,365 \mathrm{pg} / \mathrm{mL})$ and TNF-alpha $(241 \mathrm{pg} / \mathrm{mL})$.

The patient was transferred to the ICU and was managed with supportive therapy, dexamethasone $20 \mathrm{mg} / 6 \mathrm{~h}$ from day +14 , etoposide $150 \mathrm{mg} / \mathrm{m}^{2}$ on days +15 and +18 and anakinra $100 \mathrm{mg} / 12 \mathrm{~h}$ from day +15 to day +17 , with progressive improvement. Dexamethasone was slowly tapered and discontinued on day +44 (Figure 2).

Despite successful management of CAR-T related HLH, he died on day +47 due to a necrotizing pancreatitis in the context of extramedular ALL progression.

\section{METHODS}

CAR-T cell expansion in PB was monitored by multiparameter flow cytometry through detection of labeled CD19 in T cells (Human CD19 Protein $®$ ) and by quantitative PCR using the HIV /COBAS@ AmpliPrep/COBAS\& TaqManß HIV-1 Test, v2.0 (Roche, Switzerland) following manufacturer's instructions.

Tumor samples from core needle biopsies were processed using QIAamp DNA Tissue Kit (Qiagen, Germany) to detect CAR-T cells.

Plasma cytokine levels (IL-6, IL-15, IL-1 $\beta$, and GM-CSF) were determined with last generation ELISA multiplex (Ellaß, ProteinSimpleß). 
Written informed consent was obtained in compliance with our institutional review board and the Declaration of Helsinki.

\section{DISCUSSION}

HLH/MAS is an hyperinflammatory syndrome which typically consists on hyperactivation of cytotoxic T and natural killer (NK) cells and macrophages, leading to a massive cytokine production, lymphohistiocytic tissue infiltration and immune-mediated multiorgan failure ${ }^{7,9}$. CAR-T related HLH is rare, with severe and fulminant cases occurring in approximately $1 \%$ of patients receiving this treatment. However, this complication is associated with high mortality rates and a prompt diagnosis and early management is mandatory ${ }^{7}$.

Given its hyperinflammatory nature, it is considered that CRS and HLH/MAS might belong to a similar spectrum of systemic disorders, which makes HLH/MAS diagnosis difficult, especially in the context of $\mathrm{CRS}^{7}$. The traditional diagnosis criteria for secondary HLH/MAS such as HLH-2004 ${ }^{10}$ and H-Score ${ }^{11}$ are not specific, and Neelapu et al. have proposed new criteria for CAR-T related HLH/MAS, considering it is crucial to promptly diagnose this complication ${ }^{7}$. Both our patients met criteria of CAR-T related HLH/MAS according to Neelapu et al, with the second patient showing an optimal response to treatment due to its early management.

Distinction between this entity and malignancy-triggered HLH/MAS can be challenging and their management should be different. Currently, there are no generally accepted criteria for malignancy-triggered $\mathrm{HLH} / \mathrm{MAS}^{9}$. In Case 1, the presence of previous HLH/MAS at lymphoma diagnosis challenged even more this differential diagnosis. However, detection of CAR-T cells in PB and a lymph node biopsy and the paradoxical response observed in the PET-CT allowed a more specific diagnosis and management in our patient.

So far, very little has been published on CAR-T associated HLH/MAS and no formal guidelines for its management exist. Most authors recommend anti-IL6 therapy and steroids, adding etoposide if no improvement after $48 \mathrm{~h}^{7,16}$, as etoposide selectively deletes activated $\mathrm{T}$ cells and suppresses inflammatory cytokine production $^{9}$.

Anakinra, a recombinant IL-1 receptor antagonist is an emerging treatment for CAR-T associated $\mathrm{HLH} / \mathrm{MAS}^{16}$, and was used in our patients. Recent reports and preclinical studies suggest that there may be a benefit in combining Anakinra with other anti-inflammatory agents ${ }^{17}, 18$. However, due to the variability of these studies, the ideal dose schedule for this drug in HLH/MAS is still to be determined ${ }^{19}$.

Monoclonal antibodies may have a potential role in the management of this condition in the near future ${ }^{7}$. Emapalumab, an anti-IFN-gamma has been approved by the FDA for patients with refractory primary HLH/MAS and a phase two clinical trial to evaluate its efficacy in secondary HLH/MAS is undergoing, with promising interim results ${ }^{20}$. However, there is still no formal indication of Emapalumab in secondary $\mathrm{HLH} / \mathrm{MAS}^{21,22}$.

In conclusion, CAR-T cell related HLH/MAS is an unusual manifestation of severe CRS after CAR-T cell therapy, with poor prognosis, high mortality rates and a challenging diagnosis. The establishment of specific diagnosis criteria is essential for a prompt identification of patients suffering from this complication in whom any delay in treatment can be fatal. Also, the combination of diagnosis techniques for CAR-T cell follow-up allows a more precise diagnosis and more accurate distinction between CAR-T cell related or malignancy associated HLH/MAS, therefore granting a better targeted treatment. However, further studies are needed to provide better preventive and treatment strategies to improve the outcome of these patients.

\section{ACKNOWLEDGMENTS}

We would like to thank patients and their families for their collaboration towards research.

\section{AUTHOR CONTRIBUTIONS}

Conception and design: RMMR, IGC, RB, MK. 
Provision of study materials or patients: All authors.

Collection and assembly of data: RMMR, IGC, RB, MK.

Data analysis and interpretation: All authors.

Manuscript writing: RMMR, IGC, RB, MK.

Final approval of manuscript: All authors.

\section{FUNDING}

This work was partially supported by the Department of Health, Autonomous Community of Madrid grant COV20/CM, Ministry of Science and Innovation grant PID2019-107545RB-I00, Ministry of Economy and Competitiveness ISCIII-FIS grant FIS PI20/00521 co-financed by ERDF (FEDER) Funds from the European Commission, "A way of making Europe".

\section{CONFLICTS OF INTEREST}

None to declare

\section{REFERENCES}

1. Kochenderfer JN, Wilson WH, Janik JE, et al. Eradication of B-lineage cells and regression of lymphoma in a patient treated with autologous $\mathrm{T}$ cells genetically engineered to recognize CD19. Blood. 2010;116(20):4099-4102. doi:10.1182/blood-2010-04-281931

2. Maude SL, Frey N, Shaw PA, et al. Chimeric antigen receptor T cells for sustained remissions in leukemia. N Engl J Med. 2014;371(16):1507-1517. doi:10.1056/NEJMoa1407222

3. Schuster SJ, Bishop MR, Tam CS, et al. Tisagenlecleucel in Adult Relapsed or Refractory Diffuse Large B-Cell Lymphoma. N Engl J Med. 2019;380(1):45-56. doi:10.1056/NEJMoa1804980

4. Maude SL, Laetsch TW, Buechner J, et al. Tisagenlecleucel in Children and Young Adults with B-Cell Lymphoblastic Leukemia. N Engl J Med. 2018;378(5):439-448. doi:10.1056/NEJMoa1709866

5. Lee DW, Gardner R, Porter DL, et al. Current concepts in the diagnosis and management of cytokine release syndrome. Blood. 2014;124(2):188-195. doi:10.1182/blood-2014-05-552729

6. Schuster SJ, Maziarz RT, Rusch ES, et al. Grading and management of cytokine release syndrome in patients treated with tisagenlecleucel in the JULIET trial. Blood Adv. 2020;4(7):1432-1439. doi:10.1182/bloodadvances.2019001304

7. Neelapu SS, Tummala S, Kebriaei P, et al. Chimeric antigen receptor T-cell therapy - assessment and management of toxicities. Nat Rev Clin Oncol. 2018;15(1):47-62. doi:10.1038/nrclinonc.2017.148

8. Sandler RD, Tattersall RS, Schoemans H, et al. Diagnosis and Management of Secondary HLH/MAS Following HSCT and CAR-T Cell Therapy in Adults; A Review of the Literature and a Survey of Practice Within EBMT Centres on Behalf of the Autoimmune Diseases Working Party (ADWP) and Transplant Complications Working Party (TCWP). Front Immunol. 2020; 11:524. doi:10.3389/fimmu.2020.00524

9. Lehmberg K, Nichols KE, Henter JI, et al. Consensus recommendations for the diagnosis and management of hemophagocytic lymphohistiocytosis associated with malignancies. Haematologica. 2015;100(8):997-1004. doi: 10.3324/haematol.2015.123562.

10. Henter JI, Horne A, Aricó M, et al. HLH-2004: Diagnostic and therapeutic guidelines for hemophagocytic lymphohistiocytosis. Pediatr Blood Cancer. 2007;48(2):124-31. doi: 10.1002/pbc.21039.

11. Fardet L, Galicier L, Lambotte O, et al. Development and validation of the HScore, a score for the diagnosis of reactive hemophagocytic syndrome. Arthritis Rheumatol. 2014;66(9):2613-20. doi: 10.1002/art.38690.

12. Maude SL, Frey N, Shaw PA, et al. Chimeric antigen receptor $\mathrm{T}$ cells for sustained remissions in leukemia. N Engl J Med. 2014 Oct 16;371(16):1507-17. doi: 10.1056/NEJMoa1407222.

13. Yamamoto S, Matsumoto SI, Goto A, et al. Quantitative PCR methodology with a volume-based unit for the sophisticated cellular kinetic evaluation of chimeric antigen receptor T cells. Sci Rep. 2020 Oct 
21;10(1):17884. doi: 10.1038/s41598-020-74927-8.

14. Turtle CJ, Hanafi LA, Berger C, et al. CD19 CAR-T cells of defined CD4+:CD8+ composition in adult B cell ALL patients. J Clin Invest. 2016 Jun 1;126(6):2123-38. doi: 10.1172/JCI85309.

15. Demaret J, Varlet P, Trauet J, et al. Monitoring CAR T-cells using flow cytometry. Cytometry B Clin Cytom. 2021 Mar;100(2):218-224. doi: 10.1002/cyto.b.21941.

16. La Rosée P. Treatment of hemophagocytic lymphohistiocytosis in adults. Hematology Am Soc Hematol Educ Program. 2015;2015:190-6. doi: 10.1182/asheducation-2015.1.190.

17. Wohlfarth P, Agis H, Gualdoni GA, et al. Interleukin 1 Receptor Antagonist Anakinra, Intravenous Immunoglobulin, and Corticosteroids in the Management of Critically Ill Adult Patients With Hemophagocytic Lymphohistiocytosis. J Intensive Care Med. 2019;34(9):723-731. doi: $10.1177 / 0885066617711386$.

18. Strati P, Ahmed S, Kebriaei P, et al. Clinical efficacy of anakinra to mitigate CAR T-cell therapyassociated toxicity in large B-cell lymphoma. Blood Adv. 2020;4(13):3123-3127. doi: 10.1182/bloodadvances.2020002328.

19. Major A, Collins J, Craney C, et al. Management of hemophagocytic lymphohistiocytosis (HLH) associated with chimeric antigen receptor T-cell (CAR-T) therapy using anti-cytokine therapy: an illustrative case and review of the literature. Leuk Lymphoma. 2021 :1-7. doi: 10.1080/10428194.2021.1881507.

20. ClinicalTrials.gov Identifier: NCT03311854; De Benedetti et al. (EULAR 2019).

21. Locatelli F, Jordan MB, Allen C, et al. Emapalumab in Children with Primary Hemophagocytic Lymphohistiocytosis. N Engl J Med. 2020 May 7;382(19):1811-1822. doi: 10.1056/NEJMoa1911326.

22. Vallurupalli M, Berliner N. Emapalumab for the treatment of relapsed/refractory hemophagocytic lymphohistiocytosis. Blood. 2019 Nov 21;134(21):1783-1786. doi: 10.1182/blood.2019002289.

\section{FIGURES LEGENDS}

Figure 1. Evolution of CAR-T cells detection in peripheral blood by multiparameter flow cytometry (MFC) and quantitative PCR (qPCR), cytokines measurements and patient clinical management (Case 1). CAR-T cells detection is showed in cells $/ \mathrm{mL}$ and cytokines in $\mathrm{pg} / \mathrm{mL}$. Normal levels: IL-6 0.16-37.7 pg/mL; IL-1 $0.17-24 \mathrm{pg} / \mathrm{mL} ; \mathrm{IL}-15$ 1.25-13.1pg/mL; GM-CSF 0.5-728.1pg/mL.

G1 CRS: Grade 1 cytokines release syndrome; BMA: Bone marrow aspirate; Dex: Dexamethasone; Cy: Cyclophosphamide.

Figure 2. Evolution of CAR-T cells detection in peripheral blood by multiparameter flow cytometry (MFC) and quantitative PCR (qPCR), cytokines measurements and patient clinical management (Case 2). CAR-T cells detection is showed in cells $/ \mathrm{mL}$ and cytokines in $\mathrm{pg} / \mathrm{mL}$. Normal levels: IL-6 0.16-37.7 pg/mL; IL-1 0.1724pg/mL; IL-15 1.25-13.1pg/mL; GM-CSF 0.5-728.1pg/mL. G1 CRS: Grade 1 cytokines release syndrome; Dex: Dexamethasone. 


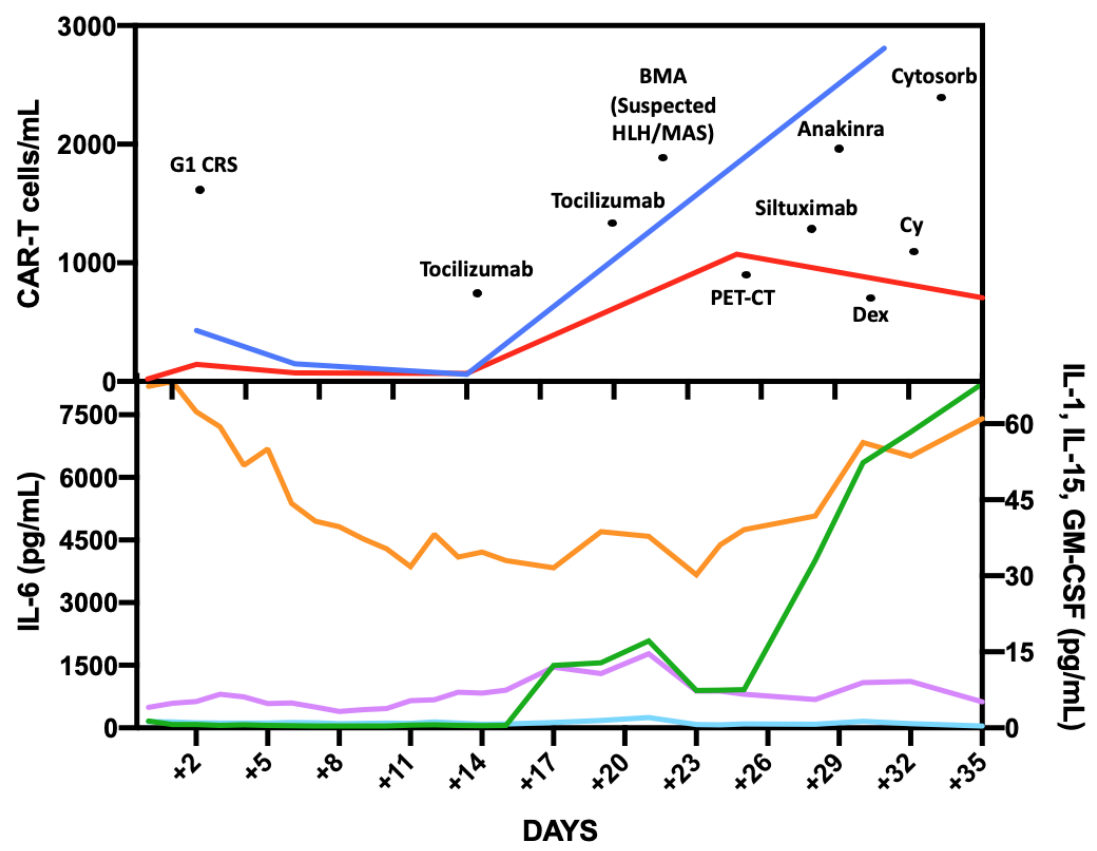

FIGURE 1. CASE 1 CAR-T CELLS AND INTERLEUKINS DYNAMICS

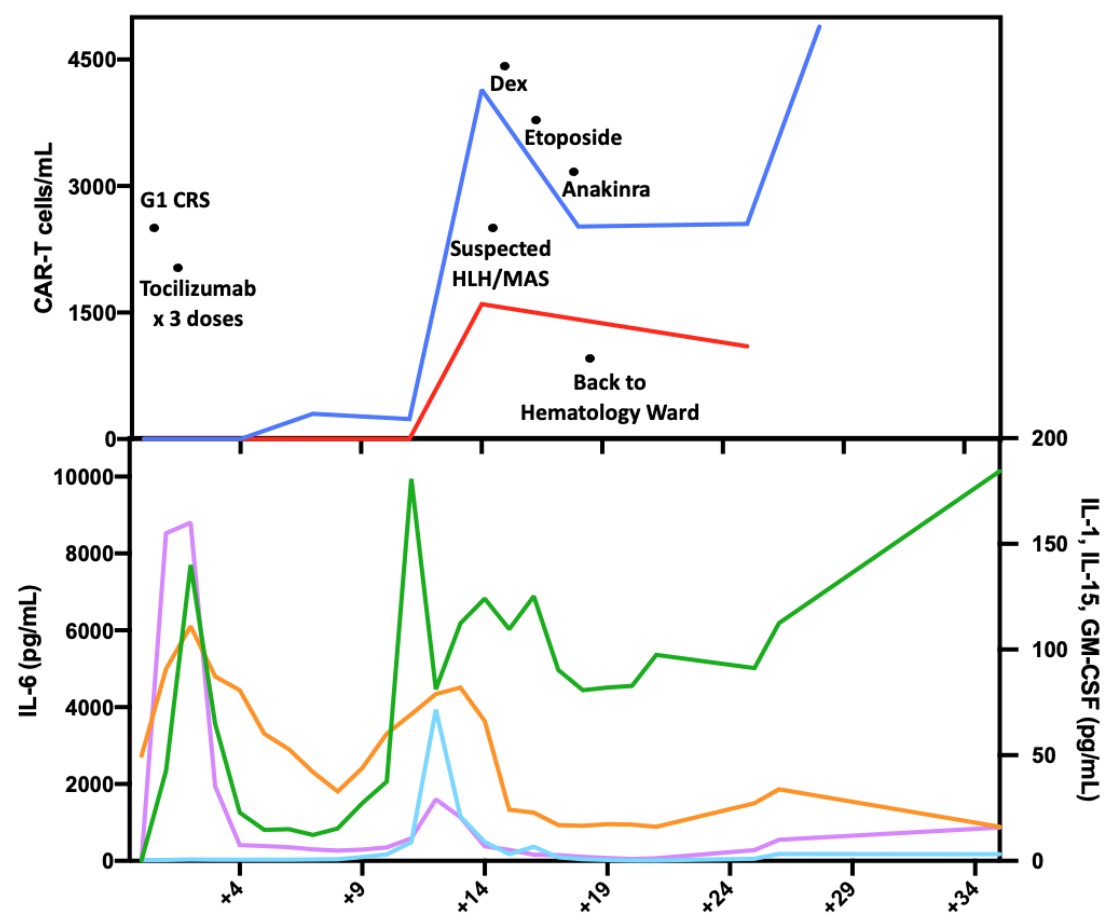

FIGURE 2. CASE 2 CAR-T CELLS AND INTERLEUKINS DYNAMICS 